\title{
Atomic-scale Imaging of Atomically-thin Organic Crystals via Low-dose STEM
}

\author{
Priti Kharel, Blanka Janicek, Sang hyun Bae and Pinshane Huang
}

University of Illinois at Urbana-Champaign, Urbana, Illinois, United States

Structural characterization is critical for the discovery and synthetic design of organic molecules with applications ranging from pharmaceutical drugs [1] to dye-sensitized-solar cells [2]. In principle, aberration-corrected scanning transmission electron microscopy (STEM) is well suited for elucidating molecular structures because techniques such as annular dark field (ADF) STEM can detect individual atoms and identify their atomic number [3]. The key challenge for imaging organic molecules via STEM is their combination of weak electron scattering and extreme beam sensitivity. For example, the critical dose of organic molecules typically ranges from 1-1000 $\mathrm{e}^{-} / \AA^{2}$ [4], several orders of magnitude lower than the doses required to image single carbon atoms in graphene. As a result, methods to extract useful information from extremely low signal-to-noise data are crucial for enabling atomic-scale imaging of organic molecules. In this work, we demonstrate the use of class averaging to reveal the structures of atomically-thin organic crystals from their low-dose ADF-STEM images with a spatial resolution of up to $2.5 \AA$.

We investigated crystals of three planar organic molecules - cobalt (II) meso-tetrakis (4-methoxyphenyl) porphyrin (CoTMPP) (Figure 1a), phthalocyanine green $(\mathrm{CuPcCl})$ (Figure 2a), and zinc phthalocyanine (ZnPc) (Figure 2e) on single-layer graphene supports. We focused on studying samples consisting of just 1-2 layers of molecules, where single images acquired below the critical dose are dominated by noise (Figure 1b). We developed methods to locate, align, and create class averages from these noisy data. As shown in Figure 1c, the class averages of 2000 frames of nominally identical regions of bilayer CoTMPP, each acquired at a dose of $51 \mathrm{e}^{-} / \AA^{2}$, allow us to visualize the metal center and the orientation of methoxyphenyl groups. For monolayer $\mathrm{CuPcCl}$ (Figure 2a-c), we resolve 16 individual chlorine atoms that decorate the edge of the molecule, consistent with previous reports [5]. Uniquely, our averages obtain contrast from the carbon atoms, visible as fluctuations in the image intensity in the region between the metal atom at the center and the chlorine atoms at the edge. For monolayer ZnPc (Figure 2e-g), we visualize the 4-fold symmetry of the molecule and remarkably distinguish the benzene rings at the edges. We quantified the effective resolution of the class averaged data, obtaining values between 2.1-2.9 (Figure 2d,h). Our results establish ADF-STEM as a promising new avenue for direct, atomic-scale imaging of organic crystals [6].
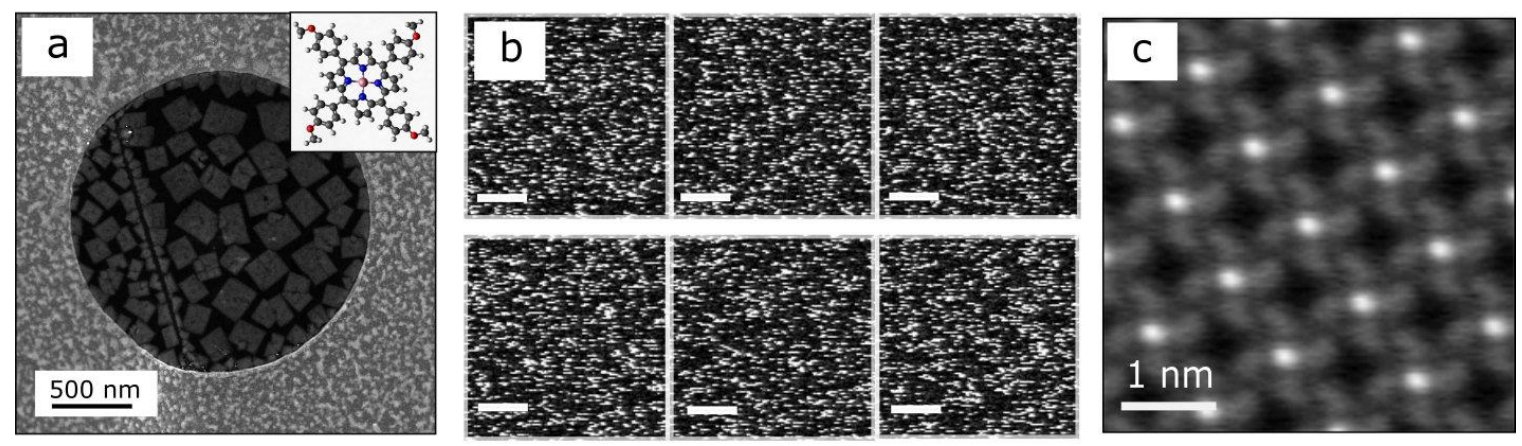

Figure 1. Class averaging for extracting structural information from noisy, low-dose images of organic molecules. (a) Low magnification ADF-STEM image showing the morphology of thermally deposited bilayer (CoTMPP) crystals on graphene. (b) High-magnification cropped sections of a raw image of a 
crystal acquired with 51 e-/Å2. Scale bars are $1 \mathrm{~nm}$. (c) Class averaged image generated by averaging 2000 frames from different nominally identical regions, as shown in (b), of the crystal.

a

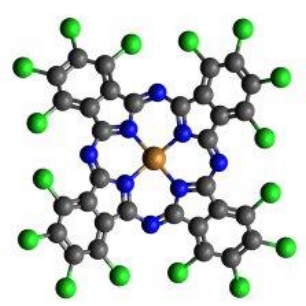

e

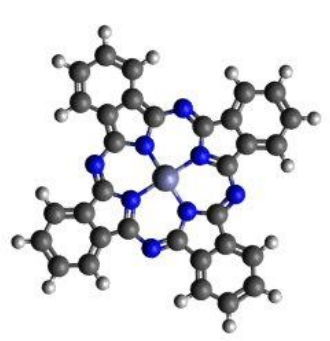

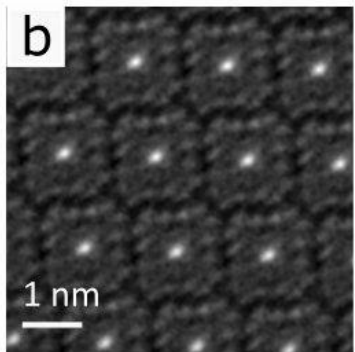
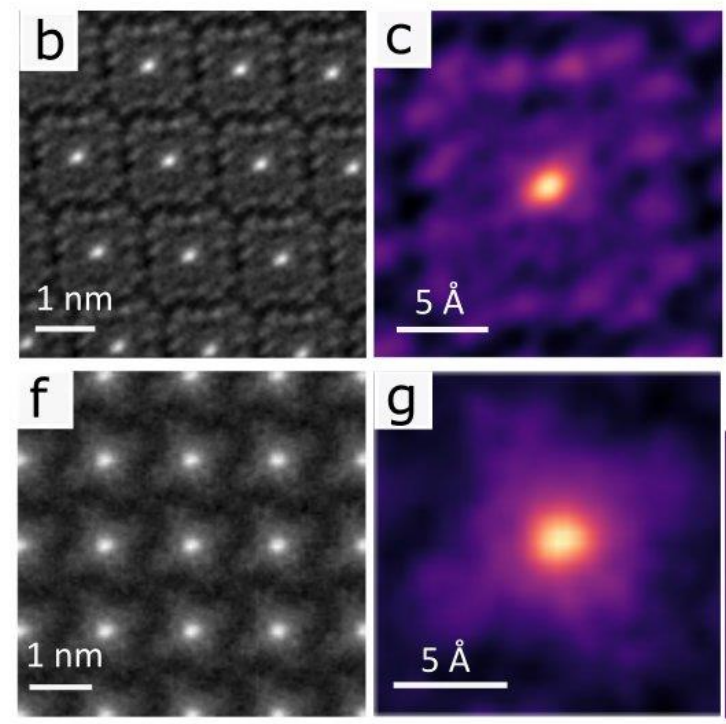
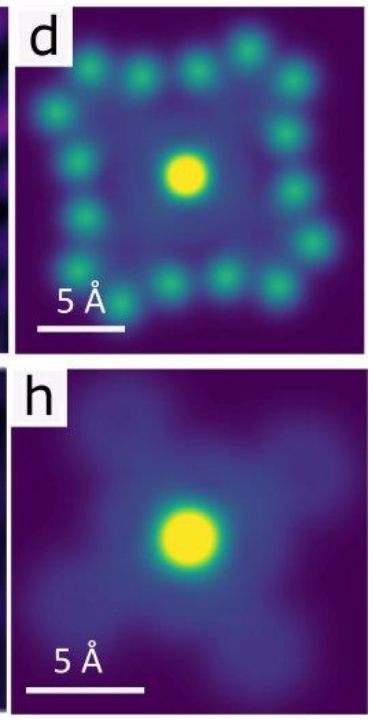

Figure 2. ADF-STEM images of phthalocyanine molecules with 2-3 $\AA$ resolution. Structures of monolayer $\mathrm{CuPcCl}$ (a) and $\mathrm{ZnPc}(\mathrm{e})$, and their respective class averaged ADF-STEM images $(\mathrm{b}, \mathrm{f})$. Smoothed class averages of single molecules $(c, g)$ are a good match for simulated ADF-STEM images (d,h) generated using a $2.5 \AA$ probe size.

\section{References}

[1] J Drews et al., Science 287 (2000), p. 1960.

[2] A Hagfeldt et al., Chem. Rev. 110 (2010), p. 6595.

[3] OL Krivanek et al., Nature 464 (2010), p. 571.

[4] RF Egerton, Micron 119 (2019), p. 72.

[5] A Mittelberger et al., Scientific Reports 8 (2018), p. 1.

[6] This work was supported by the Packard Foundation and an NSF CAREER award DMR-1846206. The experimental work was carried out in the Materials Research Laboratory. 